\title{
AVALIAÇÃO DA INFLUÊNCIA DA ADIÇÃO DE CARBONATO DE CÁLCIO NAS PROPRIEDADES MECÂNICAS DO POLIPROPILENO
}

Fernanda Miranda Torres Paiva1; Daniel Vianna Goes Araujo1; Lucas de Carvalho Cesar ${ }^{1}$,

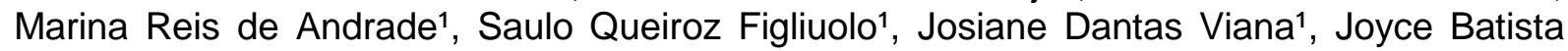
Azevedo

${ }^{1}$ Av. Orlando Gomes, 1845 - Piatã, 41650-010, Salvador/Bahia; fernanda.torres@fieb.org.br

${ }^{2}$ Rua Rui Barbosa, 710 - Centro, 44380-000, Cruz das Almas/Bahia

Resumo: A adição de cargas minerais a polímeros termoplásticos como o polipropileno (PP) esteve durante muito tempo relacionada à tentativa de redução de custos. Atualmente, com o desenvolvimento tecnológico da indústria e de novas aplicações, as cargas ganharam uma maior importância e passaram a atender mais que o objetivo econômico, por agregarem melhorias nas características físicas e químicas dos produtos. O trabalho baseia-se nessa visão e tem como objetivo a avaliação dos efeitos nas propriedades mecânicas dos compósitos formados por polipropileno (PP) e Carbonato de Cálcio (CaCO3) em concentrações de 5 e 10\%. Os resultados demostram que a adição de $\mathrm{CaCO}_{3}$, proporciona uma diminuição na capacidade de deformação do compósito, confirmada pelo aumento no módulo de elasticidade e diminuição da deformação específica na ruptura, além de diminuição da resistência ao impacto. Sugere-se novas pesquisas utilizando nanopartículas de carbonato de cálcio para verificar o comportamento da matriz polimérico frente a elementos de menor tamanho.

Palavras-Chave: Polipropileno; carbonato de cálcio; ensaios mecânicos destrutivos.

\section{EVALUATION OF THE INFLUENCE OF THE ADDITION OF CALCIUM CARBONATE ON MECHANICAL PROPERTIES OF POLYPROPYLENE}

\begin{abstract}
The addition of mineral charge to thermoplastic polymers such as polypropylene (PP) has long been related to the attempt to reduce costs. Nowadays, with the technological development of the industry and new applications, the loads have gained more importance and started to serve more than the economic objective, by adding improvements in the physical and chemical characteristics of the products. This work is based on this view and aims to evaluate the effects on the mechanical properties of composites formed by polypropylene (PP) and calcium carbonate $\left(\mathrm{CaCO}_{3}\right)$ in concentrations of 5 and $10 \%$. The results show that the addition of $\mathrm{CaCO}_{3}$, provides a decrease in the deformability of the composite, confirmed by the increase
\end{abstract}


in elastic modulus and decrease of the specific deformation at rupture, as well as decreased impact strength. Further research using calcium carbonate nanoparticles is suggested to verify the behavior of the polymeric matrix against smaller elements.

Keywords: polypropylene; calcium carbonate; destructive mechanical tests. 


\section{INTRODUÇÃO}

A palavra polímero vem do grego poli (muitos) + meros (iguais), os polímeros são compostos macromoleculares, formados pela repetição de uma estrutura química mais simples denominada mero e que pode ter origem orgânica ou inorgânica. Estes materiais são os mais utilizados na indústria e possuem alta aplicabilidade comercial, tais como: embalagens, adesivos, componentes automotivos, circuitos integrados e vestimentas. [1-6]

Os polímeros podem ser classificados em termoplásticos ou termorrígidos. Os termoplásticos são materiais que podem ser moldados quando submetidos à temperatura e pressão, retiradas as solicitações de pressão e temperatura, esses componentes se solidificam e assumem o formato desejado, são solúveis, possuem cadeia linear ou ramificada e como exemplo temos o polietileno, polipropileno e o PVC. Os termorrígidos, também chamados de termofixos, são materiais que quando submetidos à temperatura e pressão amolecem e fluem adquirindo a forma do molde, reagem quimicamente formando ligações cruzadas entre cadeias e se solidificam. Novas variações de temperatura e pressão não geram influência nesses elementos, tornando-os materiais insolúveis e não-recicláveis, como exemplo dessa classe temos a resina de fenol-formaldeído (baquelite) e epóxi (araldite) [1-4]

O polipropileno é utilizado tanto no processo de extrusão como na moldagem por injeção, o material é sintetizado pela polimerização de propileno, um monômero derivado de produtos petrolíferos e se tornou um dos mais importantes polímeros devido à sua versatilidade e satisfatórias propriedades mecânicas aliadas ao baixo custo. Os principais minerais utilizados como cargas em polímeros são: talco, pirofilita, calcita, dolomita, caulinita, esmectita, muscovita, quartzo, wollastonita e barita, porem o carbonato de cálcio tornou-se o mineral mais empregado nas indústrias de papel e plástico. O mineral pode ser obtido da exploração de diversas jazidas, dentre as quais se destacam, as de calcita, calcáreo e mármore, dependendo do grau de moagem do carbonato de cálcio obtém-se cargas com diferentes tamanhos de partículas, tamanhos menores são os mais desejáveis em função das melhores propriedades mecânicas e acabamento superficial [5-7].

A adição de cargas minerais a polímeros termoplásticos como o polipropileno (PP) esteve durante muito tempo relacionada à tentativa de redução de custos. Atualmente, com o desenvolvimento tecnológico da indústria e de novas aplicações, as cargas ganharam uma maior importância e passaram a atender mais que o objetivo econômico, por agregarem melhorias nas características físicas e químicas dos produtos [6-8].

Este trabalho baseia-se nessa visão e tem como objetivo a avaliação dos efeitos nas propriedades mecânicas dos compósitos formados por polipropileno (PP) e Carbonato de Cálcio (CaCO3) em concentrações de 5 e 10\%

\section{METODOLOGIA}

Para realização do estudo da influência da carga de carbonato de cálcio (fase dispersa) em polipropileno (matriz), foram preparados compósitos com diferentes concentrações de carga. Os materiais foram processados através de uma extrusora 


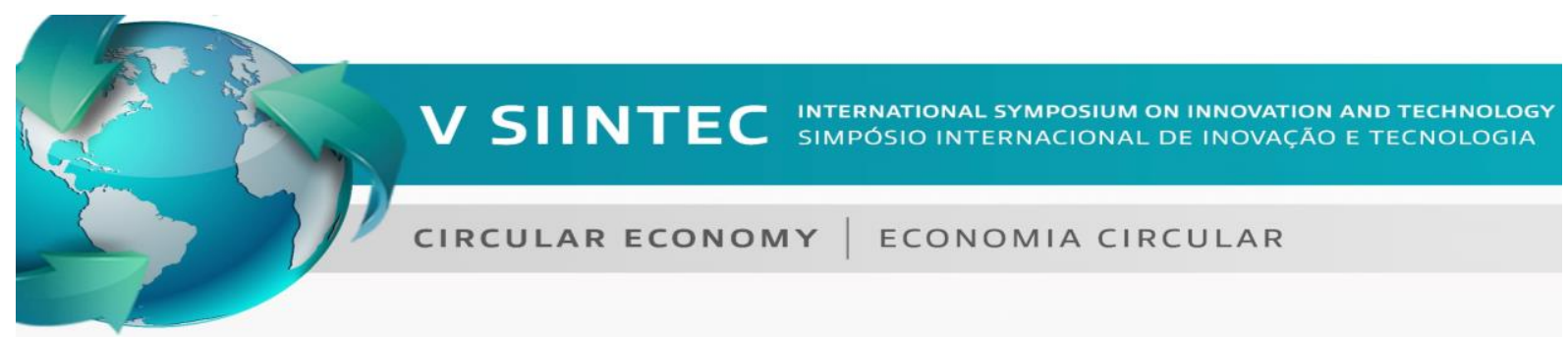

da marca Imacon, modelo DRC 30:40 IF (Figura 1), com configuração dupla rosca corotacional, de diâmetro $30 \mathrm{~mm}$ e razão $L / D$ de 40 . No processo de extrusão foram gerados três tipos de amostras: a) polipropileno puro (PP); b) Polipropileno com 5\% de Carbonato de Cálcio (PP+5\%CaCO3); c) Polipropileno com 10\% de Carbonato de Cálcio (PP+10\%CaCO3). Utilizou-se como parâmetros de processamento a velocidade de rotação de $70 \mathrm{rpm}$ e um perfil de rosca com média intensidade de mistura.

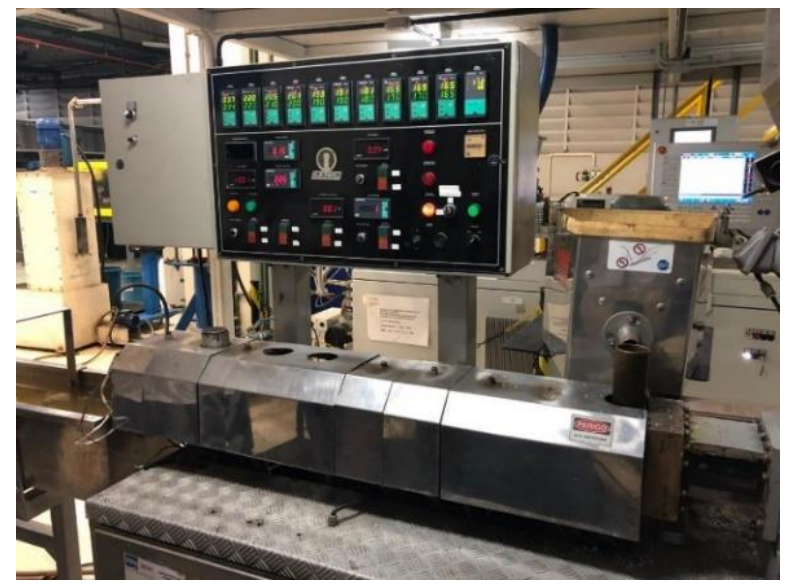

Figura 1 - Extrusora dupla rosca co-rotacional da marca Imacon.

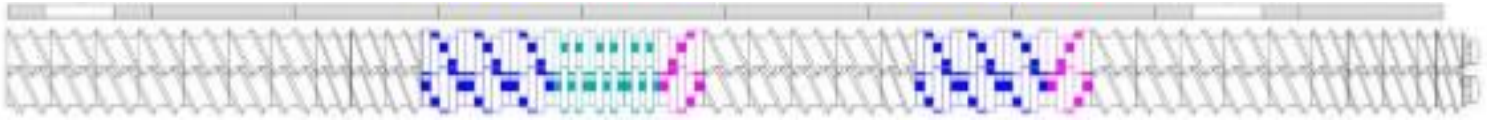

Figura 2 - Configuração do perfil de rosca utilizado na produção dos compósitos (Software WinTXS).

Os compósitos obtidos pelo processo de extrusão, com formato de filamento (Figura 3), seguiram para trituradora (Figura 4). Em seguida, o material foi seco em estufa por um período de $8 \mathrm{~h}$ a uma temperatura de $100^{\circ} \mathrm{C}$ para garantir a qualidade dos corpos de prova posteriormente processados.

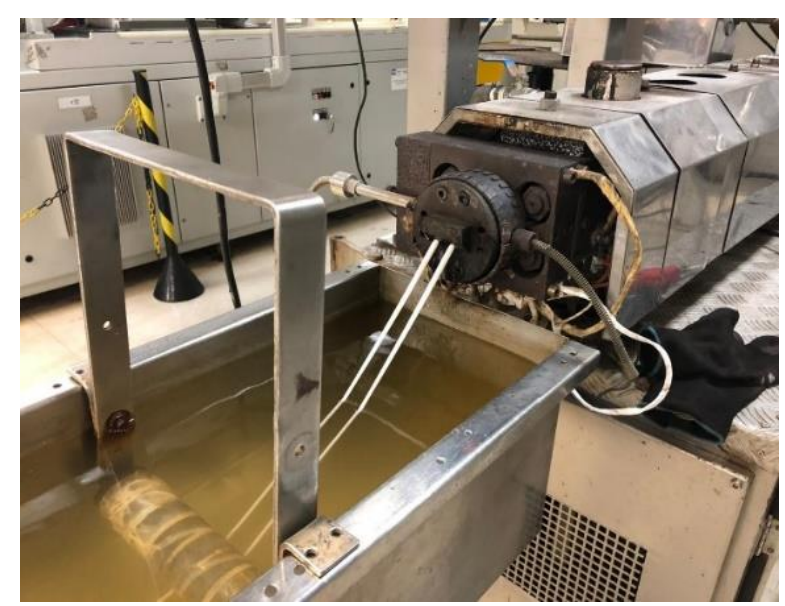

Figura 3 - Extrusão de filamento do compósito.

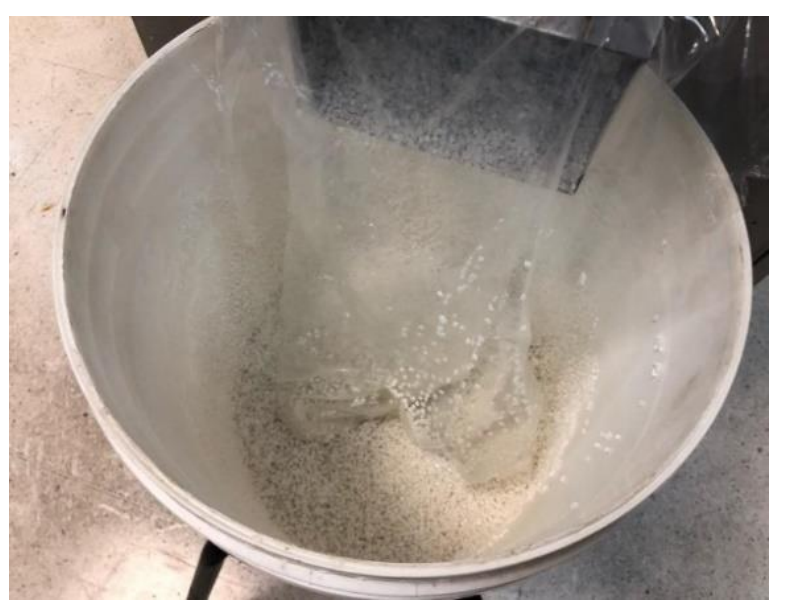

Figura 4 - Compósito triturado.

\subsection{PREPARAÇÃO DOS CORPOS DE PROVA}


Utilizou-se a injetora da marca ROMI, modelo Primax 100, com força de fechamento de 100 toneladas (Erro! Fonte de referência não encontrada.). Os corpos de prova foram preparados com auxílio de ferramentas de injeção previamente manufaturadas para obtenção de peças com formato de gravata segundo as normas ISO 527 para ensaio de tração [09] e a ISO 180 para ensaio de impacto [10]. Após o processo de injeção, todos os corpos de prova passaram por inspeção visual para identificar a presença de falhas.

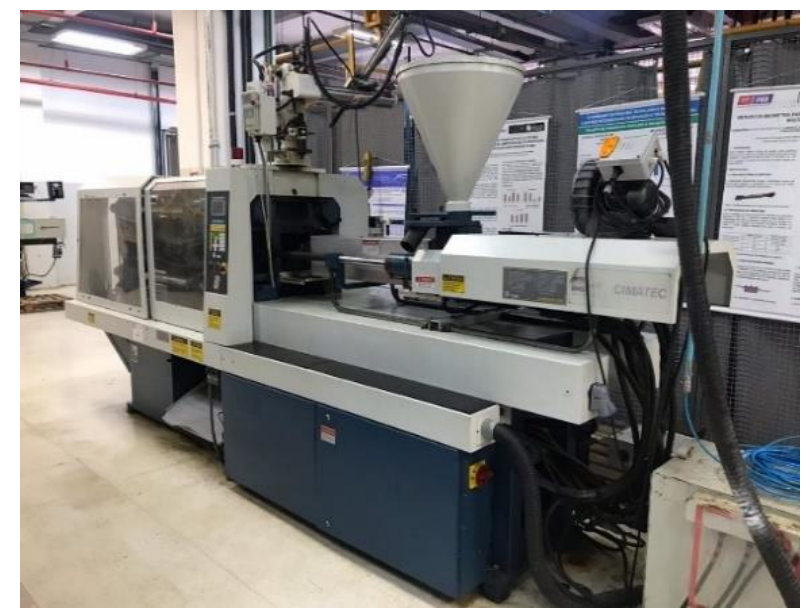

Figura 5 - Máquina injetora utilizada no procedimento.

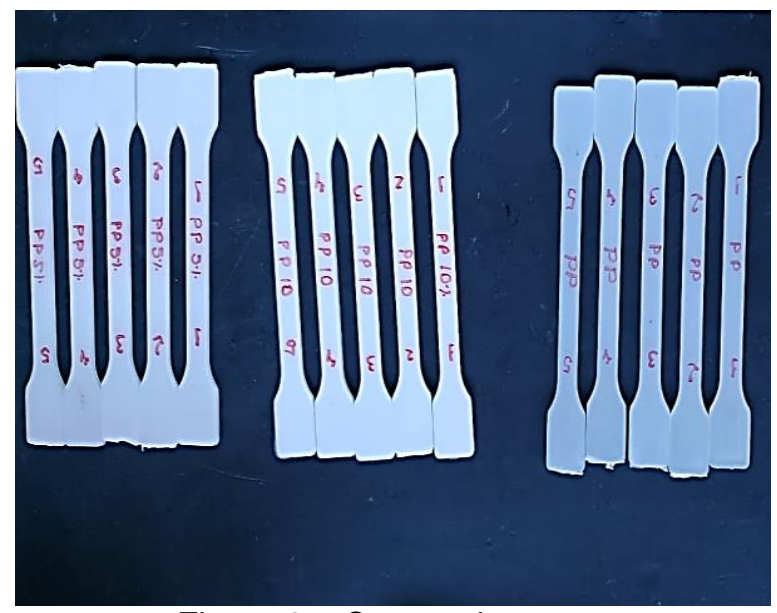

Figura 6 - Corpos de prova.

\subsection{CARATERIZAÇÃO DOS MATERIAIS}

As propriedades do polipropileno e do carbonato de cálcio utilizados no estudo estão apresentadas nas Tabelas 1 e 2.

Tabela 1. Propriedades do polipropileno EP 440L da empresa Braskem.

\begin{tabular}{l|c}
\hline \multicolumn{2}{c}{ EP 440L } \\
\hline Propriedades de controle & Valor \\
\hline İndice de Fluidez $\left(230^{\circ} \mathrm{C} / 2,16 \mathrm{Kg}\right)$ & $6 \mathrm{~g} / 10 \mathrm{~min}$ \\
\hline Propriedades Típicas & 0,895 \\
\hline Densidade $\left(\mathrm{g} / \mathrm{cm}^{3}\right)$ & 1050 \\
\hline Módulo de Flexão Secante a 1\% (MPa) & 24 \\
\hline Resistência à Tração no Escoamento $(\mathrm{MPa})$ & 6 \\
\hline Alongamento no escoamento $(\%)$ & 60 \\
\hline Dureza Rockwell (Escala R) & $\mathrm{NB}$ \\
\hline Resistência ao impacto Izod a 23으 $(\mathrm{J} / \mathrm{m})$ & 75 \\
\hline Resistência ao impacto Izod a $-20^{\circ} \mathrm{C}(\mathrm{J} / \mathrm{m})$ & 85 \\
\hline Temperatura de Deflexão Térrnica a 0,455 & \\
MPa (ํㅡ) & \\
\hline
\end{tabular}

Tabela 2. Propriedades do carbonato de cálcio Mícron 1/9CB da empresa MICRON-ITA

\begin{tabular}{|c|c|}
\hline \multicolumn{2}{|l|}{ Mícron 1/9CB } \\
\hline Propriedades Físicas & Valor \\
\hline Tamanho mediano (mícron) & Max. 2,0 \\
\hline Corte Superior a 97\% (mícron) & Max. 7,5 \\
\hline $\mathrm{pH}\left(1 \mathrm{~g} / 100 \mathrm{~g} \mathrm{H}_{2} \mathrm{O}\right)$ & 9 \\
\hline Teor Abrasivo $\left(\mathrm{g} / \mathrm{m}^{2}\right)$ & 57 \\
\hline Índice de Absorção (DOP) (g/100g) & 40 \\
\hline Coloração & Branca \\
\hline Alvura (\%) & 98 \\
\hline Densidade aparente $\left(\mathrm{g} / \mathrm{cm}^{3}\right)$ & 0,95 \\
\hline Resíduo em malha 400 (\%) & $<0,005$ \\
\hline Resíduo em malha 500 (\%) & $<0,01$ \\
\hline \multicolumn{2}{|l|}{ Propriedades Químicas } \\
\hline $\mathrm{CaCO}_{3}(\%)$ & Min. 83 \\
\hline $\mathrm{MgCO}_{3}(\%)$ & Máx. 15 \\
\hline Insolúveis em $\mathrm{HCl}(\%)$ & $\leq 1,5$ \\
\hline $\mathrm{R}_{2} \mathrm{O}_{3}(\%)$ & $\leq 0,5$ \\
\hline Umidade (\%) & $\leq 0,3$ \\
\hline $\mathrm{SiO}_{2}(\%)$ & $\leq 0,75$ \\
\hline
\end{tabular}

Os materiais foram caracterizados por: Ensaio de tração: Realizados seguindo a norma ISO 527 [9], com o auxílio de uma máquina universal de ensaios de tração 
EMIC DL 2000. Os dados foram obtidos a partir da média dos resultados de cinco amostras de cada um dos materiais; Ensaio de impacto IZOD: Foram realizados seguindo a norma ISO 180 [10], com o auxílio de um pêndulo de impacto EMIC - AIC, com martelo de 2,7J. Os dados foram obtidos a partir da média dos resultados de cinco amostras de cada um dos materiais; Caracterização Morfológica: Utilizou-se microscópio eletrônico de varredura (MEV) da marca Jeol, modelo JSM-6510LV. As amostras foram preparadas através do processo de metalização a vácuo.

\section{RESULTADOS E DISCUSSÃO}

A Tabela 3 apresenta os valores médios e seus respectivos desvios padrões da tensão máxima, deformação específica na ruptura e módulo de elasticidade. Analisando os valores da deformação específica na ruptura, foi possível constatar uma tendência na diminuição dos valores com o aumento dos percentuais de carga mineral, porém na amostra PP $+5 \%$ CaCO3 houve uma anomalia comprovada pelo elevado desvio padrão, o que aponta um possível erro durante a preparação da amostra. Os resultados demostram que a adição de $\mathrm{CaCO}_{3}$, proporciona uma diminuição na capacidade de deformação do compósito, essa afirmação pode ser confirmada pela tendência no aumento do módulo de elasticidade e diminuição da deformação específica na ruptura proporcionais ao acréscimo dos teores de carbonato de cálcio. Este comportamento é esperado e comprovado em outros estudos, visto que a inserção da carga aumenta a rigidez do polímero e dificulta o movimento de cisalhamento entre as moléculas da matriz [6-8].

Tabela 3. Resultados dos ensaios de tração.

\begin{tabular}{c|c|c|c|c|c|c}
\hline \multirow{2}{*}{ Materiais } & \multicolumn{2}{|c|}{ Tensão Máxima } & \multicolumn{2}{c}{$\begin{array}{c}\text { Deformação específica } \\
\text { na ruptura }\end{array}$} & \multicolumn{2}{c}{ Módulo de Elasticidade } \\
\cline { 2 - 6 } & $(\mathrm{MPa})$ & $\mathrm{DP}$ & $(\%)$ & $\mathrm{DP}$ & $(\mathrm{MPa})$ & $\mathrm{DP}$ \\
\hline Polipropileno & 27,26 & 2,69 & 197,90 & 66,10 & 846,70 & 370,10 \\
\hline $\mathrm{PP}+5 \% \mathrm{CaCO} 3$ & 28,85 & 0,60 & 245,20 & 125,20 & 885,00 & 382,40 \\
\hline $\mathrm{PP}+10 \% \mathrm{CaCO} 3$ & 27,57 & 0,44 & 108,20 & 53,58 & 990,60 & 349,30 \\
\hline
\end{tabular}

A Tabela 4, apresenta os valores médios de energia de ruptura e os desvios padrões obtidos nos ensaios de impacto das amostras. Observa-se uma diminuição da resistência ao impacto com a adição de carbonato de cálcio. Isto se deve ao aumento de rigidez do compósito causada pela adição de carga mineral, o material passa a absorver menos energia durante o impacto e fratura com mais facilidade $[6,8]$.

Tabela 4. Resultados dos ensaios de impacto.

\begin{tabular}{c|c|c}
\hline Material & E (J) & Desvio Padrão \\
\hline Polipropileno (PP) & 0,512 & 0,030 \\
\hline $\mathrm{PP}+5 \% \mathrm{CaCO}_{3}$ & 0,404 & 0,036 \\
\hline $\mathrm{PP}+10 \% \mathrm{CaCO}_{3}$ & 0,468 & 0,036 \\
\hline
\end{tabular}




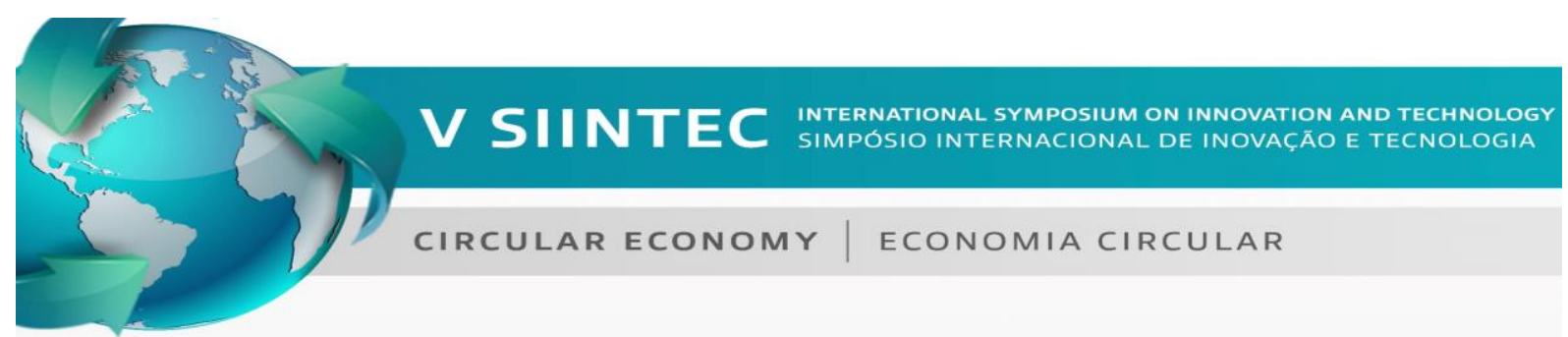

As Figuras 7 e 8 apresentam imagens obtidas por Microscopia Eletrônica de Varredura (MEV) da superfície de fratura dos corpos de prova do composto PP $+10 \%$ $\mathrm{CaCO}_{3}$ utilizados nos ensaios de impacto. Constata-se nas imagens uma dispersão uniforme da carga (fase mais clara) por toda a matriz polimérica (fase mais escura), as partículas de carbonato de cálcio apresentam um formato esférico com diâmetro médio em torno de $2 \mu \mathrm{m}$ e observa-se uma boa aderência das partículas de $\mathrm{CaCO}_{3}$ com a matriz polimérica sem grandes presenças de vazios.

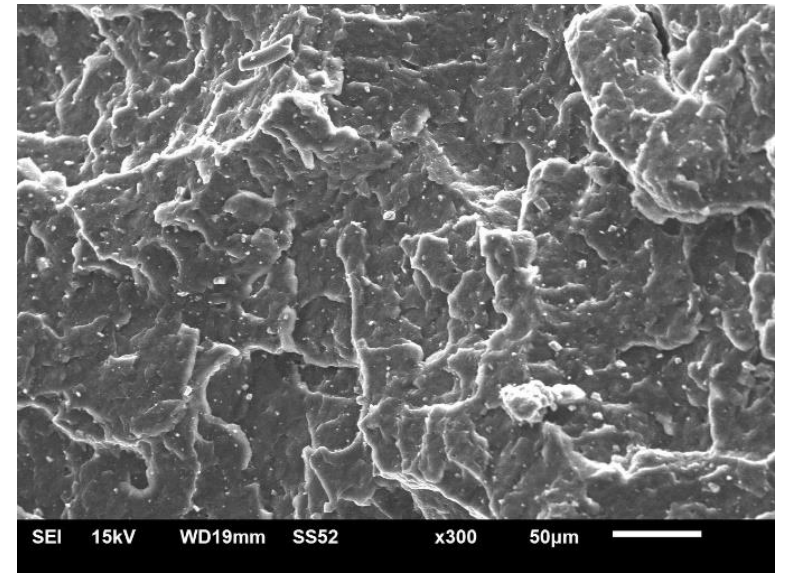

Figura 7 - Imagem por MEV do composto PP + $10 \% \mathrm{CaCO}_{3}$ aumentada $300 x$

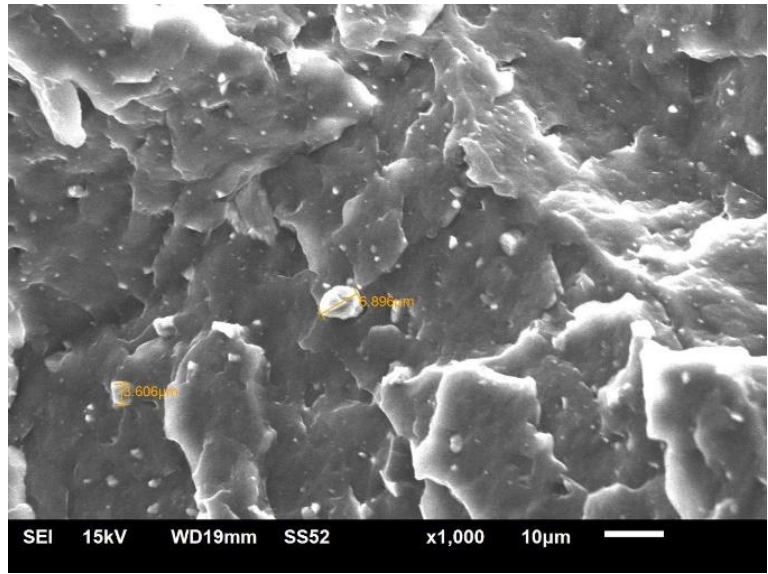

Figura 8 - Imagem por MEV do composto PP + $10 \% \mathrm{CaCO}_{3}$ aumentada $1000 \mathrm{x}$

O tamanho das partículas do carbonato de cálcio são importantes nas caracteristicas mecânicas do material, pois partículas menores e consequentemente maior superfície de contato, influenciam diretamente na adesão interfacial do compósito. Em outros estudos realizados com objetivo de analisar a influencia mecânica da adição de nanopartículas de carbonato de cálcio em matrizes de polipropileno, os pesquisadores apontaram um aumento no módulo de elasticidade acompanhado de um aumento em torno de $300 \%$ na resistencia ao impacto do compósito [11-14].

\section{CONCLUSÃO}

A utilização da carga mineral em polipropileno apresentou modificações significativas nas características mecânicas do compósito, os resultados apontam que a adição de $\mathrm{CaCO}_{3}$, proporciona uma diminuição da capacidade de deformação do compósito e redução na tenacidade do material, sugere-se novas pesquisas utilizando nanopartículas de carbonato de cálcio para verificar o comportamento da matriz polimérico frente a elementos de menor tamanho.

\section{REFERÊNCIAS}


${ }^{1}$ CANEVAROLO Jr. S. V. Ciência dos polímeros: um texto básico para tecnólogos e engenheiros. São Paulo: Artliber, 2002.

${ }^{2}$ CALLISTER, W. D; RETHWISCH, D. G. Ciência e Engenharia de Materiais - Uma Introdução. São Paulo: LTC, 2016.

${ }^{3}$ SIMÊNCIA, E. C. A. Ciência dos Materiais. Londrina: Editora e Distribuidora Educacional S.A., 2016.

${ }^{4}$ WASILKOSKI, C. L. Comportamento mecânico dos materiais poliméricos. Tese de doutorado. Curitiba: UFPR, 2006.

${ }^{5}$ LIMA, A. B.T. Aplicações de cargas minerais em polímeros. Dissertação de Mestrado. São Paulo: USP, 2007

${ }^{6}$ SILVA, E. A. Processamento e caracterização de compósitos de polipropileno/carbonato de cálcio ( $\mathrm{PP} / \mathrm{CaCO}$ ) para possível utilização na indústria automobilística. Dissertação de Mestrado. Volta Redonda: UniFOA, 2017. ${ }^{7}$ GORGAL, C. J. P; SCNEIDER, C. G; BARBOSA, R. V; NETO, R. B. Comparação de uso de Carbonato de Cálcio em compósitos utilizando uma matriz de Polipropileno com diferentes IFs. Anais $8^{\circ}$ Congresso Brasileiro de Polimeros, p. 220-22, São Paulo, 2005.

${ }^{8}$ MIGUEL, J. J. P. Estudo Comparativo das Propriedades Térmicas e Mecânicas do Polipropileno Reforçado com Óxido de Zinco, Carbonato de Cálcio e Talco. Dissertação de Mestrado. Salvador: SENAI CIMATEC, 2010.

$9 \quad$ IISO 527-1 - Plastics - Determination of tensile properties - Part 1: General principles, 2019.

${ }^{10}$ _. ISO 180 - Plastics - Determination of Izod impact strength, 2000.

${ }^{11}$ EIRAS, D; PESSAN, L. A. Mechanical properties of polypropylene/calcium carbonate nanocomposites. Materials Research, v. 12, n. 4, p. 517-522, 2009

${ }^{12}$ FUAD, M. Y. A; HANIM, H; ZARINA, R; MOHD ISHAK, Z. A; HASSAN, A. Polypropylene/calcium carbonate nanocomposites - effects of processing techniques and maleated polypropylene compatibiliser. Polymer Letters, v. 04, n. 10, p. 611-620, 2010.

${ }^{13} \mathrm{CHAN}, \mathrm{C} . \mathrm{M}$; WU, J; LI, J. X; CHEUNG, Y. K. Polypropylene/calcium carbonate nanocomposites. Polymer, v. 43, n. 10, p. 2981-2992, 2002.

${ }^{14}$ YANG, K; YANG, Q; LI, G; SUN, Y; FENG, D. Morphology and mechanical properties of polypropylene/calcium carbonate nanocomposites. Materials Letters, v. 60, n. 6, p. 805-809, 2006. 